\title{
Selection of reference genes for RT-qPCR analysis in tumor tissues from male hepatocellular carcinoma patients with hepatitis B infection and cirrhosis
}

\author{
Shuang Liu ${ }^{\mathrm{a}, 1}$, Pengfei Zhu ${ }^{\mathrm{b}, 1}$, Ling Zhang ${ }^{\mathrm{c}}$, Shanlong Ding ${ }^{\mathrm{b}}$, Sujun Zheng ${ }^{\mathrm{a}}$, Yang Wang $^{\mathrm{a}}$ and \\ Fengmin $\mathrm{Lu}^{\mathrm{b}, *}$ \\ ${ }^{a}$ Beijing Artificial Liver Treatment and Training Center, Beijing Youan Hospital, Capital Medical University, \\ Beijing, China \\ ${ }^{\mathrm{b}}$ Department of Microbiology and Infectious Disease Center, Peking University Health Science Center, Beijing, \\ China \\ ${ }^{\mathrm{c}}$ Henan Cancer Hospital, Zhengzhou, Henan, China
}

\begin{abstract}
.
BACKGROUND: Reverse transcription quantitative real-time polymerase chain reaction (RT-qPCR) has been widely used to quantify relative gene expression because of the high specificity, sensitivity and accuracy of this technique. However, its reliability is strongly depends on the expression stability of reference gene used for data normalization. Therefore, identification of reliable and condition specific reference genes is critical for the success of RT-qPCR.

OBJECTIVE: Hepatitis B virus (HBV) infection, male gender and the presence of cirrhosis are widely recognized as the leading independent risk factors for the development of hepatocellular carcinoma (HCC). This study aimed to select reliable reference gene for RT-qPCR analysis in HCC patients with all of those risk factors.

METHODS: Six candidate reference genes were analyzed in 33 paired tumor and non-tumor tissues from untreated HCC patients. The genes expression stabilities were assessed by geNorm and NormFinder.

RESULTS: C-terminal binding protein 1(CTBP1) was the most stable gene among the 6 candidate genes evaluated by both geNorm and NormFinder. The expression stability values were 0.08 for CTBP1 and UBC, 0.09 for HPRT1, 0.12 for HMBS, 0.14 for GAPDH and 0.18 for $18 \mathrm{~S}$ with geNorm analysis. The stability values suggested by NormFinder software were CTBP1: 0.044, UBC: 0.063, HMBS: 0.072, HPRT1: 0.072, GAPDH: 0.098 and 18S rRNA: 0.161.

CONCLUSION: This is the first systematic analysis which suggested CTBP1 as the highest expression-stable gene in human male HBV infection related-HCC with cirrhosis. We recommend CTBP1 as the best candidate reference gene when RT-qPCR was used to determine gene(s) expression in HCC. This may facilitate the relevant HBV related HCC studies in the future.
\end{abstract}

Keywords: Hepatocellular carcinoma, hepatitis B virus, liver cirrhosis, male gender, C-terminal binding protein 1, reference gene, RT-qPCR

\footnotetext{
${ }^{1}$ Equal contribution.

* Corresponding author: Fengmin Lu, Department of Microbiology and Infectious Disease, Peking University Health Science Center, Beijing 100191, China. Tel.: +86 010 82805136; Fax: +86 010 82805136; E-mail: Lu.fengmin@hsc.pku.edu.cn.
} 


\section{Introduction}

The technique of quantitative real-time reverse transcription polymerase chain reaction (RT-qPCR) has provided a powerful tool for quantifying gene expression. RT-qPCR is characterized with both higher sensitivity and specificity, as compared with traditional mRNA analysis [1,2]. However, the accuracy of RTqPCR is influenced by a number of variables, such as RNA stability, quantity, purity, enzymatic efficiency in cDNA synthesis and PCR amplification [3]. Thus, it is extremely important to use highly reliable reference gene to normalize the experiments. The most commonly used measure for normalization is to use one or several housekeeping genes as reference gene in experiment. Glyceraldehyde-3-phosphate dehydrogenase (GAPDH) has been widely used as internal control for RT-qPCR analysis in human hepatocellular carcinoma (HCC) [4]. However, several recent lines of evidences implicate GAPDH has also been involved in some physiological functions, such as tRNA transport [5], translational control [6], binding with viral RNAs [7]. Moreover, increased GAPDH expression has been reported in breast cancer, renal cell carcinoma, lung cancer, prostate carcinoma and HCC [811]. Separate studies even suggested that GAPDH expression was correlated with chemotherapy-induced DNA damage response in vitro [12-14]. A study even suggested the heavily regulation of GAPDH during HCC carcinogenesis and progression in patients with HBV related HCC [15], as it can affect the proliferative potential of cancer cells in HCC [16]. HBV infection, the presence of cirrhosis and male gender are repeatedly recognized as the independent risk factors for the development of HCC [17-19]. Therefore, it is worthwhile to select an appropriate reference gene for RT-qPCR analysis in HCC patients with those high risk factors. In present study, 6 commonly used reference genes (CTBP1, UBC, HPRT1, HMBS, GAPDH and 18s RNA) were investigated by RT-qPCR in 33 paired tumor and non-tumor tissues from male un-treated HCC patients with evidence of chronic HBV infection and cirrhosis. The expression stability of these genes was assessed by both geNorm and NormFinder softwares.

\section{Materials and methods}

\subsection{Patients information and sample collection}

From January to April, 2013, we have collected 33 paired tumor and adjuvant non-tumor tissues from
HCC patients in the Henan Cancer Hospital (Zhengzhou, China). The selected HCC patients fulfilled the following entire criterion: seropositive for HBV surface antigen (HBsAg) or HBV DNA positive in tumor tissues, receiving no chemotherapy before surgery, male gender and with liver cirrhosis. Liver cirrhosis and HCC were diagnosed by the experienced pathologists.

The paired tumor and non-tumor tissues were collected when patients underwent hepatectomy. Then specimens were immediately snap-frozen in liquid nitrogen. The study protocol was approved by the Ethic Committee of the Henan Cancer Hospital.

\subsection{Analysis of $m R N A$ expression by $R T-q P C R$}

The tissue specimens were ground in liquid nitrogen and homogenized in Trizol (Invitrogen, Carlsbad, CA) by mortar. Total RNA was extracted according to the manufacturer's instruction. Genomic DNA contamination was removed by the on-column digestion with the RNase-free DNase (TaKaRa Biotechnology, Japan). The concentrations of the isolated total RNA were measured at $260 \mathrm{~nm}$ to $280 \mathrm{~nm}$ with NanoDrop ND-2000 spectrophotometer (NanoDrop Technologies, Wilmington, DE, USA). The threshold inclusion values for the RNA samples were $>1.90$ for the A260/A280 ratio and $28 \mathrm{~S} / 18 \mathrm{~S}$ ratio $\geqslant 1.7$. The integrity of RNA samples was confirmed by electrophoresis on a $1 \%$ agarose gel.

First-strand cDNA was synthesized using Random Primer according to the manufacturer's instructions (Revert Aid First strand cDNA Synthesis Kit, Fermentas, Lithuania). Primers for RT-qPCR assays of CTBP1, HPRT1, HMBS, 18S rRNA, GAPDH and UBC were designed using Primer Premier 5.0 (Table 1). The Roche LightCycler 480 detection system (Roche Diagnostics, Germany) was used for real-time PCR instruments. Reactions were prepared in a total volume of $20 \mu \mathrm{L}$ containing $10 \mu \mathrm{L}$ of SYBR Green master mix (Roche Diagnostics, Germany), $0.5 \mu \mathrm{L}$ of each $10 \mu \mathrm{M}$ primer $(500 \mathrm{nM}), 1 \mu \mathrm{L}$ cDNA, and $8 \mu \mathrm{L}$ nuclease-free sterile water. All standards and samples were run in triplicate on 96-well reaction plates. The cycle conditions were set as follows: start with $10 \mathrm{~min}$ template denaturation at $95^{\circ} \mathrm{C}, 40$ cycles of denaturation at $95^{\circ} \mathrm{C}$ for $30 \mathrm{~s}$, and elongation at $72^{\circ} \mathrm{C}$ for $30 \mathrm{~s}$. This cycle was followed by a melting curve analysis, baseline and cycle threshold values ( $\mathrm{Ct}$ values) were automatically determined for all plates using Roche LightCycler 480 Software. Ct values difference be- 
Table 1

Six candidate reference genes

\begin{tabular}{|c|c|c|c|c|}
\hline Gene symbol & Gene name & Primer sequence $\left(5^{\prime} \rightarrow 3^{\prime}\right)$ & Accession no. & Amplicon size (bp) \\
\hline \multirow[t]{2}{*}{ HMBS } & hydroxymethylbilane synthase & F1: CCCTGGAGAAGAATGAAGTGGA & NM_000190.3 & $133 \mathrm{bp}$ \\
\hline & & R1: TTTGGGTGAAAGACAACAGCATC & & \\
\hline \multirow[t]{2}{*}{ HPRT1 } & hypoxanthine phosphoribosyltransferase 1 & F1: TTGCTGACCTGCTGGATTACAT & NM_000194.2 & $262 b p$ \\
\hline & & R1: CTTGCGACCTTGACCATCTTTG & & \\
\hline \multirow[t]{2}{*}{ CTBP1 } & C-terminal Binding Protein 1 & F1: TTCACCGTCAAGCAGATGAGAC & NM_001012614.1 & $156 b p$ \\
\hline & & R1: CTGGCTAAAGCTGAAGGGTTCC & & \\
\hline \multirow[t]{2}{*}{ 18S rRNA } & $18 \mathrm{~S}$ ribosomal RNA & F1: GGTGGAGCGATTTGTCTGGTTA & NR_003286.2 & $167 \mathrm{bp}$ \\
\hline & & R1: CGGACATCTAAGGGCATCACAG & & \\
\hline \multirow[t]{2}{*}{ UBC } & Ubiquitin $\mathrm{C}$ & F1: CCCTTCTCGGCGATTCTG & NM_021009.4 & $155 \mathrm{bp}$ \\
\hline & & R1: CATTGTCAAGTGACGATCACAGC & & \\
\hline \multirow[t]{2}{*}{ GAPDH } & glyceraldehyde-3-phosphate dehydrogenase & F:TGTTCCAATATGATTCCACCC & NM_002046.3 & $185 \mathrm{bp}$ \\
\hline & & R:CTTCTCCATGGTGCGTGAAGA & & \\
\hline
\end{tabular}
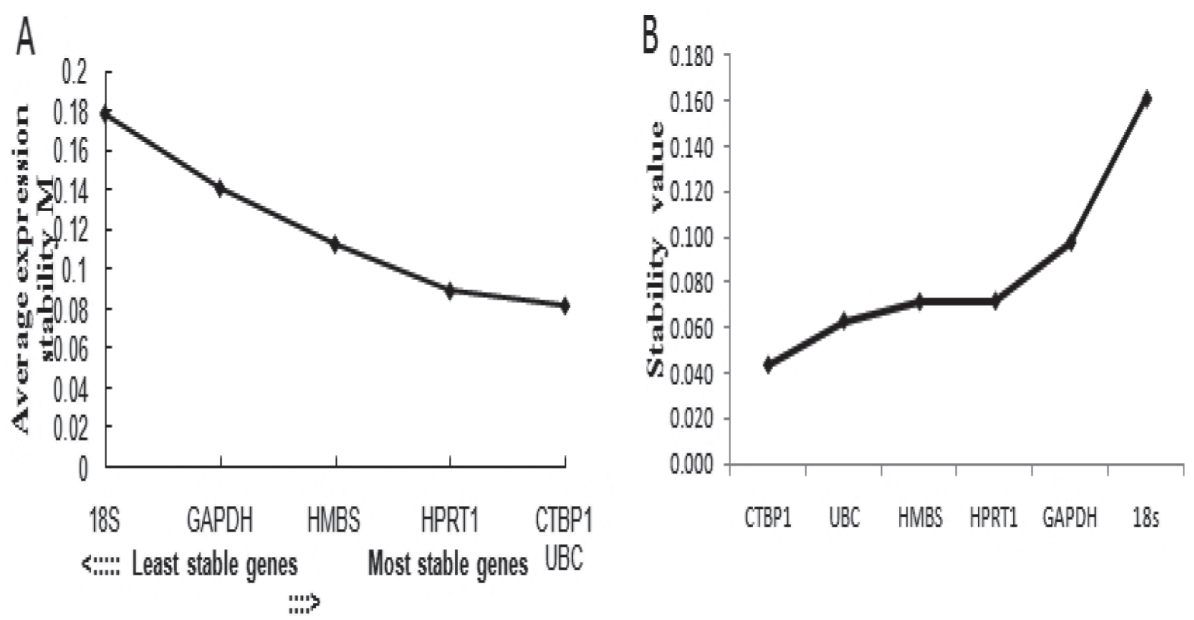

Fig. 1. Relative expression stability of 6 candidate reference genes evaluated by geNorm software (A) and NormFinder program (B). A. Low 'M value' correlates to higher gene expression stability. M values of CTBP1 and UBC, HPRT1, HMBS, GAPDH and 18S rRNA are as follows: The expression stability values were $0.08,0.09,0.12,0.14$ and 0.18 geNorm analysis; B. Low 'Stability Value' correlates to higher gene expression stability. Stability values of CTBP1, UBC, HMBS, HPRT1, GAPDH and 18S rRNA was as follows: 0.044, 0.063, 0.072, 0.072, 0.098, 0.161. Both geNorm and Normfinder software showed that CTBP1 was the most stable gene.

tween triplicates no more than 1 was considered acceptable and used for average $\mathrm{Ct}$ values calculation. For data set, those genes that had greater than twofold increase $\left(2^{-\Delta C t}>2\right)$ or decrease $\left(2^{-\Delta C t}<0.5\right)$ of expression were considered for differential expression, or will be defined as equal expression $\left(0.5 \leqslant 2^{-\Delta C t} \leqslant\right.$ $2)$, as previously reported [20].

\subsection{Candidate reference genes selection by geNorm and NormFinder}

Data were analyzed by two broadestly used softwares geNorm (version 3.5) [3] and NormFinder [21]. GeNorm calculates an expression stability value (M value) for each candidate gene based on pairwise comparisons of variability. The $\mathrm{M}$ value calculated for each gene is used to rank genes from least to most stable. The M-value of 1.5 was set as cut-off value of an endogenous control gene, as the authors suggested [22]. The NormFinder software is available at http://www.mdl.dk. It can calculate the stability values of each candidate reference genes for normalization. A low stability value means a low combined intraand inter-group variation proves high expression stability [21]. Using these two softwares, the most stable gene is calculated.

\subsection{Statistical analyses}

Student's t test or wilcoxon rank test was used for statistical analyses with SAS software (version 9.1, SAS Institute, Inc., 2004), $P \leqslant 0.05$ was considered statistically significant. Results were the means \pm SEM of 3 separate experiments. 


\section{Results}

The Candidate Gene Expression Profile in Paired Tumor and Non Tumor Tissues Analyzed by geNorm.

The expression stabilities (M value) of overall candidate reference genes ranged from 0.08 to 0.18 . High $\mathrm{M}$ values indicated the highly variable and less stably expression of the genes. On the other hand, genes with low $M$ values suggested the stable expression and low variability. Based on data obtained, the $18 \mathrm{~S}$ was the least stable reference gene with $\mathrm{M}$ value of 0.18 , GAPDH was better than $18 \mathrm{~S}$, but CTBP1 and UBC were the two most stable genes with the $M$ value of 0.08 (Fig. 1A).

The Candidate Gene Expression Profile in Paired Tumor and Non Tumor Tissues Analyzed by NormFinder.

Meanwhile, the expression stabilities of overall candidate reference genes were analyzed by NormFinder. Again, CTBP1 was also the most stable gene. GAPDH, UBC, HMBS and the $18 \mathrm{~S}$ was the subsequently less stable reference gene as the above demonstrated (Fig. 1B).

Therefore, the two software programs together suggest CTBP1 as the most expression-stable gene in HBV-related HCC, either in tumorous or in nontumorous tissues, or both. In contrast, the commonly used GAPDH is not as stable as CTBP and may not be suitable to be used as the reference gene for RT-qPCR analysis when quantificationally measuring a gene's expression in HCC tissues.

\section{Dissusion}

About 600000 people die every year due to the acute or chronic consequences of hepatitis $\mathrm{B}$, including liver failure, cirrhosis and HCC [25]. Presently about $80 \%$ of HCC patients have chronic HBV infection in China. Cirrhosis due to chronic hepatitis B is also one of the leading risk factor for HCC. The other upcoming risk factors include male gender, old age, obesity, diabetes and related nonalcoholic fatty liver disease [1719]. Although the risk factors are clear, the underlying mechanisms remain largely elusive. The change of genes expression profiles in HCC may provide clues for the hunting of key genetic event relevant to $\mathrm{HCC}$ development. RT-qPCR has been widely used to quantify the relative gene expression. However, the most challenging part of real-time PCR data analysis is identifying suitable housekeeping genes stably expressed to normalize among all of the samples. GAPDH was most frequently used as a reference gene for RT-qPCR analysis in HCC [4]. However, several recent studies supporting revealed the increased GAPDH expression in both mRNA and protein levels in HCC [15,23,24]. So selection of suitable reference gene to instead of GAPDH for RT-qPCR data analysis in HCC patients is in an urgent need. Cirrhosis due to chronic hepatitis B and male gender are two well known leading risk factors for HCC. Therefore we investigated 6 generally used housekeeping genes as candidate genes in tumor tissues from 33 male HCC patients with evidence of chronic HBV infection and the presence of cirrhosis.

The geNorm and NormFinder are two well-known softwares, each with different strategies to evaluate reference genes $[21,22]$. To ensure getting reliable result, both softwares were employed in this study. The raw $\mathrm{Ct}$ values were analyzed by both software in parallel.

The gene expression stability spectrums were somehow different when evaluated by the two analysis methods. Using geNorm analysis the expression stabilities is evaluated by $M$ value. The $M$ value ranged from 0.08 to 0.18 . CTBP1 and UBC ranked as the best gene, and then it was HPRT1, HMBS, GAPDH, and 18S. Interestingly, CTBP1 was the most stable gene evaluated by the NormFinder software as well, UBC, HMBS, HPRT1, GAPDH and the $18 \mathrm{~S}$ subsequently was the less stable reference gene. Therefore, based on the both software programs CTBP1 exhibited the highest expression stability for RT-qPCR analysis in untreated HCC tumorous and non-tumorous tissues for male patients with $\mathrm{HBV}$ infection and cirrhosis. In contrast, GAPDH was only better than $18 \mathrm{~S}$, but worse than CTBP1, HPRT1 and UBC. As to our knowledge, it is the first systemic study of reference genes in cohort composed of male HCC patients with underlying HBV infection and cirrhosis.

In conclusion, the current study reveals CTBP1 as the most suitable and reliable reference genes for RTqPCR data analysis in HCC patients with underlying HBV infection and cirrhosis. This result may facilitate HBV related HCC study in the future.

\section{Acknowledgement}

This study was supported by the Project for the Major Infectious Diseases 2012ZX10002005 from Ministry of Science and Technology of the People's Republic of China. 


\section{References}

[1] Heid CA, Stevens J, Livak KJ, Williams PM: Real time quantitative PCR. Genome Res 1996, 6(10): 986-994.

[2] Gibson UE, Heid CA, Williams PM: A novel method for real time quantitative RT-PCR. Genome Res 1996, 6(10): 9951001.

[3] Vandesompele J, De Preter K, Pattyn F, Poppe B, Van Roy N, De Paepe A, Speleman F: Accurate normalization of realtime quantitative RT-PCR data by geometric averaging of multiple internal control genes. Genome Biol 2002, 3(7): RESEARCH0034.

[4] Matsumura M, Ijichi M, Shiratori Y, Togo G, Hikiba Y, Inoued K, Kohara M, Omata M: Simple quantitative assay of alphafetoprotein mRNA in liver tissue using the real-time detection polymerase chain reaction assay - its application for clinical use. Hepatol Res 2001, 20(1): 84-96.

[5] Sirover MA: New insights into an old protein: The functional diversity of mammalian glyceraldehyde-3-phosphate dehydrogenase. Biochim Biophys Acta 1999, 1432(2): 159-184.

[6] Yi M, Schultz DE, Lemon SM: Functional significance of the interaction of hepatitis A virus RNA with glyceraldehyde 3-phosphate dehydrogenase (GAPDH): opposing effects of GAPDH and polypyrimidine tract binding protein on internal ribosome entry site function. J Virol 2000, 74(14): 6459-6468.

[7] Lin SS, Chang SC, Wang YH, Sun CY, Chang MF: Specific interaction between the hepatitis delta virus RNA and glyceraldehyde 3-phosphate dehydrogenase: an enhancement on ribozyme catalysis. Virology 2000, 271(1): 46-57.

[8] Vila MR, Nicolas A, Morote J, de I, Meseguer A: Increased glyceraldehyde-3-phosphate dehydrogenase expression in renal cell carcinoma identified by RNA-based, arbitrarily primed polymerase chain reaction. Cancer 2000, 89(1): $152-164$

[9] Tokunaga K, Nakamura Y, Sakata K, Fujimori K, Ohkubo M, Sawada K, Sakiyama S: Enhanced expression of a glyceraldehyde-3-phosphate dehydrogenase gene in human lung cancers. Cancer Res 1987, 47(21): 5616-5619.

[10] Revillion F, Pawlowski V, Hornez L, Peyrat JP: Glyceraldehyde-3-phosphate dehydrogenase gene expression in human breast cancer. Eur J Cancer 2000, 36(8): 1038-1042.

[11] Harada N, Yasunaga R, Higashimura Y, Yamaji R, Fujimoto $\mathrm{K}$, Moss J, Inui H, Nakano Y: Glyceraldehyde-3-phosphate dehydrogenase enhances transcriptional activity of androgen receptor in prostate cancer cells. J Biol Chem 2007, 282(31): 22651-22661.

[12] Meyer-Siegler K, Mauro DJ, Seal G, Wurzer J, DeRiel JK, Sirover MA: A human nuclear uracil DNA glycosylase is the 37-kDa subunit of glyceraldehyde-3-phosphate dehydrogenase. Proc Natl Acad Sci U S A 1991, 88(19): 8460-8464.

[13] Azam S, Jouvet N, Jilani A, Vongsamphanh R, Yang X, Yang S, Ramotar D: Human glyceraldehyde-3-phosphate dehydro- genase plays a direct role in reactivating oxidized forms of the DNA repair enzyme APE1. J Biol Chem 2008, 283(45): 30632-30641.

[14] Krynetski EY, Krynetskaia NF, Bianchi ME, Evans WE: A nuclear protein complex containing high mobility group proteins B1 and B2, heat shock cognate protein 70, ERp60, and glyceraldehyde-3-phosphate dehydrogenase is involved in the cytotoxic response to DNA modified by incorporation of anticancer nucleoside analogues. Cancer Res 2003, 63(1): 100106.

[15] Gao Q, Wang XY, Fan J, Qiu SJ, Zhou J, Shi YH, Xiao YS, Xu Y, Huang XW, Sun J: Selection of reference genes for realtime PCR in human hepatocellular carcinoma tissues. J Cancer Res Clin Oncol 2008, 134(9): 979-986.

[16] Ganapathy-Kanniappan S, Kunjithapatham R, Geschwind JF: Glyceraldehyde-3-phosphate dehydrogenase: A promising target for molecular therapy in hepatocellular carcinoma. Oncotarget, 3(9): 940-953.

[17] Lee MH, Yang HI, Liu J, Batrla-Utermann R, Jen CL, Iloeje UH, Lu SN, You SL, Wang LY, Chen CJ: Prediction models of long-term cirrhosis and HCC risk in chronic hepatitis B patients: Risk scores integrating host and virus profiles. Hepatology.

[18] Yang HI, Sherman M, Su J, Chen PJ, Liaw YF, Iloeje UH, Chen CJ: Nomograms for risk of hepatocellular carcinoma in patients with chronic hepatitis B virus infection. J Clin Oncol, 28(14): 2437-2444

[19] Mittal S, El-Serag HB: Epidemiology of Hepatocellular Carcinoma: Consider the Population. J Clin Gastroenterol.

[20] Claverie JM: Computational methods for the identification of differential and coordinated gene expression. Human molecular genetics 1999, 8(10): 1821-1832.

[21] Andersen CL, Jensen JL, Orntoft TF: Normalization of realtime quantitative reverse transcription-PCR data: A modelbased variance estimation approach to identify genes suited for normalization, applied to bladder and colon cancer data sets. Cancer Res 2004, 64(15): 5245-5250.

[22] Thellin O, Zorzi W, Lakaye B, De Borman B, Coumans B, Hennen G, Grisar T, Igout A, Heinen E: Housekeeping genes as internal standards: use and limits. J Biotechnol 1999, 75(23): 291-295.

[23] Gong Y, Cui L, Minuk GY: Comparison of glyceraldehyde3-phosphate dehydrogenase and 28s-ribosomal RNA gene expression in human hepatocellular carcinoma. Hepatology 1996, 23(4): 734-737.

[24] Sun W, Xing B, Sun Y, Du X, Lu M, Hao C, Lu Z, Mi W, Wu $\mathrm{S}$, Wei $\mathrm{H}$ et al.: Proteome analysis of hepatocellular carcinoma by two-dimensional difference gel electrophoresis: novel protein markers in hepatocellular carcinoma tissues. Mol Cell Proteomics 2007, 6(10): 1798-1808.

[25] Don Ganem, M.D, and Alfred M. Prince, M.D. Hepatitis B Virus Infection - Natural History and Clinical Consequences. N Engl J Med 2004; 350: 1118-1129. 